International Mathematical Forum, Vol. 9, 2014, no. 35, 1725 - 1739

HIKARI Ltd, www.m-hikari.com

http://dx.doi.org/10.12988/imf.2014.410170

\title{
Alternate Locations of Equilibrium Points and Poles in Complex Rational Differential Equations
}

\author{
Koh Katagata \\ National Institute of Technology, Ichinoseki College \\ Takanashi, Hagisho, Ichinoseki, Iwate 021-8511 Japan \\ Copyright (c) 2014 Koh Katagata. This is an open access article distributed under the \\ Creative Commons Attribution License, which permits unrestricted use, distribution, and \\ reproduction in any medium, provided the original work is properly cited.
}

\begin{abstract}
We study configurations of simple equilibrium points of first order complex differential equations consisting of the iteration of rational functions. Rational functions which we deal with have the unit circle or the extended real line as Julia sets. Properties of Julia sets and the EulerJacobi formula lead to alternate locations of equilibrium points and poles of the complex differential equations.
\end{abstract}

Mathematics Subject Classification: 37C10, 32A10, 37F10

Keywords: Equilibrium points, The Euler-Jacobi formula, Julia sets

\section{Introduction}

Let $D$ be a domain in $\mathbb{C}$ and let $f: D \rightarrow \mathbb{C}$ be a holomorphic function. We consider the first order differential equation

$$
\dot{z} \equiv \frac{d z}{d t}=f(z),
$$

where $t \in \mathbb{R}$ and $z \in D$. A point $\zeta \in D$ is an equilibrium point of the differential equation (DE) if it satisfies that $f(\zeta)=0$. Let $f(x+i y)=$ $u(x, y)+i v(x, y)$. We identify the differential equation (DE) with the system of differential equations

$$
\dot{x}=u(x, y), \quad \dot{y}=v(x, y)
$$


in $\mathbb{R}^{2}$. Equilibrium points are categorized as stable nodes, unstable nodes, centers, stable foci, unstable foci and saddles. In general, in order to classify equilibrium points of a system of the differential equations

$$
\dot{x}=\varphi(x, y), \quad \dot{y}=\psi(x, y)
$$

in $\mathbb{R}^{2}$, we have to consider the linearization of the map $(x, y) \mapsto(\varphi(x, y), \psi(x, y))$ and the behavior of solutions of the system of the differential equations $(*)$ near equilibrium points. However, the behavior of the solutions of the differential equation (DE) near equilibrium points is well-known. We can classify equilibrium points complex analytically as follows.

Theorem $1.1([1,2])$. Let $\zeta \in D$ be an equilibrium point of the differential equation (DE). Then the equilibrium point $\zeta$ is

(1) a stable node if and only if $f^{\prime}(\zeta)<0$,

(2) an unstable node if and only if $f^{\prime}(\zeta)>0$,

(3) a center if and only if $\operatorname{Im} f^{\prime}(\zeta) \neq 0$ and $\operatorname{Re} f^{\prime}(\zeta)=0$,

(4) a stable focus if and only if $\operatorname{Im} f^{\prime}(\zeta) \neq 0$ and $\operatorname{Re} f^{\prime}(\zeta)<0$,

(5) an unstable focus if and only if $\operatorname{Im} f^{\prime}(\zeta) \neq 0$ and $\operatorname{Re} f^{\prime}(\zeta)>0$.

Besides, the differential equation (DE) does not have saddles.

In [3], the author have studied the complex differential equation

$$
\dot{z}=f_{c}^{* n}(z) \equiv f_{c}^{\circ n}(z)-z,
$$

where

$$
f_{c}(z)=\frac{1+c}{2(1-c)}\left(z-\frac{1}{z}\right) \text { with } c \in[0,1 / 3]
$$

and $f_{c}^{\circ n}=f_{c} \circ \cdots \circ f_{c}$ is the $n$-th iteration of $f_{c}$. Configurations of equilibrium points of the complex differential equation (DE: $c ; n)$ are as follows.

Theorem 1.2 ([3]). For all positive integers $n$, the following statements hold.

(a) In the case that $0 \leq c<1 / 3$, the number of equilibrium points of $(\mathrm{DE}: c ; n)$ on $\mathbb{C} \backslash \mathbb{R}$ is exactly two and the two equilibrium points are stable nodes.

(b) In the case that $c=1 / 3$, there are no equilibrium points of (DE:c;n) on $\mathbb{C} \backslash \mathbb{R}$. 
(c) All equilibrium points of the differential equation (DE:c;n) are symmetric with respect to the real axis.

(d) Every equilibrium points of $(\mathrm{DE}: c ; n)$ on the real axis are unstable nodes.

(e) Equilibrium points of $(\mathrm{DE}: c ; n)$ on the real axis and poles of $f_{c}^{* n}$ are located alternately.

Considering properties of the complex differential equation (DE $: c ; n)$ and the Julia set of the rational function $f_{c}$, we can obtain the analogical results to Theorem 1.2.

Throughout this paper, we consider two rational functions $A$ and $B$. The rational function $B$ is a Blaschke product

$$
B(z)=e^{2 \pi i \theta} z \frac{z-a_{1}}{1-\overline{a_{1}} z} \frac{z-a_{2}}{1-\overline{a_{2}} z} \cdots \frac{z-a_{\nu}}{1-\overline{a_{\nu}} z}
$$

where $a_{j} \in \mathbb{D}=\{z \in \mathbb{C}:|z|<1\}$ for $1 \leq j \leq \nu$ and $\theta \in[0,1)$. The rational function $A$ is a conjugate of $B$, namely

$$
A(z)=\phi \circ B \circ \phi^{-1}(z), \phi(z)=i \frac{z+1}{z-1} .
$$

Moreover, we deal with the differential equation

$$
\dot{z}=A^{* n}(z) \equiv A^{\circ n}(z)-z, \quad(\mathrm{DE}: \boldsymbol{a} ; \theta ; n)
$$

where $\boldsymbol{a}=\left(a_{1}, \ldots, a_{\nu}\right) \in \mathbb{D}^{\nu}$ and $A^{\circ n}=A \circ \cdots \circ A$ is the $n$-th iteration of $A$. Let $\mathcal{E P}\left(A^{* n}\right)$ be the set of all equilibrium points of $A^{* n}$. Our main result is the following.

Main Theorem . For any $\boldsymbol{a}=\left(a_{1}, \ldots, a_{\nu}\right) \in \mathbb{D}^{\nu}, \theta \in[0,1)$ and $n \geq 1$, the following statements hold.

(a) Equilibrium points of $(\mathrm{DE}: \boldsymbol{a} ; \theta ; n)$ on $\mathbb{C} \backslash \mathbb{R}$ are $\pm i$ and the two equilibrium points are stable nodes or stable foci.

(b) All equilibrium points of the differential equation (DE:a; $\theta ; n$ ) are symmetric with respect to the real axis.

(c) Every equilibrium points of (DE: $\boldsymbol{a} ; \theta ; n)$ on the real axis are unstable nodes.

(d) Equilibrium points of (DE: $\boldsymbol{a} ; \theta ; n)$ on the real axis and poles of $A^{* n}$ are located alternately. 


\section{Dynamics of rational functions $A$ and $B$}

Let $f: \hat{\mathbb{C}} \rightarrow \hat{\mathbb{C}}$ be a rational function. The rational function $f$ can be written as

$$
f(z)=\frac{p(z)}{q(z)}
$$

where $p$ and $q$ are polynomials with no common roots. The rational function $f$ is continuous with respect to the spherical metric. The degree $\operatorname{deg}(f)$ of $f$ is the maximum of the degrees of $p$ and $q$. The degree $\operatorname{deg}\left(f^{\circ n}\right)$ of $f^{\circ n}$ is equal to $(\operatorname{deg}(f))^{n}$.

Definition 2.1. Let $f: \hat{\mathbb{C}} \rightarrow \widehat{\mathbb{C}}$ be a non-constant rational function. The Fatou set $\mathcal{F}(f)$ of $f$ is defined as

$\mathcal{F}(f)=\left\{z \in \hat{\mathbb{C}}:\right.$ the family $\left\{f^{\circ n}\right\}_{n=1}^{\infty}$ is normal in some open neighborhood of $\left.z\right\}$.

The Julia set $\mathcal{J}(f)$ of $f$ is the complement $\mathcal{J}(f)=\hat{\mathbb{C}} \backslash \mathcal{F}(f)$. The Fatou set $\mathcal{F}(f)$ is open and the Julia set $\mathcal{J}(f)$ is closed.

We suppose that $d=\operatorname{deg}(f) \geq 2$. Here are some basic properties of the Fatou set and the Julia set.

- The Fatou set and the Julia set are completely invariant, namely

$$
f(\mathcal{F}(f))=\mathcal{F}(f)=f^{-1}(\mathcal{F}(f)) \text { and } f(\mathcal{J}(f))=\mathcal{J}(f)=f^{-1}(\mathcal{J}(f)) .
$$

- For a positive integer $n$,

$$
\mathcal{F}\left(f^{\circ n}\right)=\mathcal{F}(f) \text { and } \mathcal{J}\left(f^{\circ n}\right)=\mathcal{J}(f) .
$$

- The Julia set $\mathcal{J}(f)$ is non-empty.

- The Julia set $\mathcal{J}(f)$ has no isolated point.

- The Julia set $\mathcal{J}(f)$ is the smallest closed completely invariant set containing at least three points.

- If the Julia set $\mathcal{J}(f)$ has an interior point, then $\mathcal{J}(f)=\hat{\mathbb{C}}$.

- If the Julia set $\mathcal{J}(f)$ is disconnected, it has uncountably many components.

- The Fatou set $\mathcal{F}(f)$ has either zero, one, two or countably many components. 
Let $z_{0}$ be a point in $\hat{\mathbb{C}}$. The point $z_{0}$ is a periodic point of $f$ if there exists a positive integer $n$ such that $f^{\circ n}\left(z_{0}\right)=z_{0}$. Such the smallest $n$ is called the period of $z_{0}$. The point $z_{0}$ is a fixed point of $f$ if the period of $z_{0}$ is one.

Definition 2.2. Let $z_{0}=f^{\circ n}\left(z_{0}\right)$ be a periodic point of period $n$. The multiplier $\lambda=\lambda\left(z_{0}\right)$ at $z_{0}$ is defined as

$$
\lambda= \begin{cases}\left(f^{\circ n}\right)^{\prime}\left(z_{0}\right) & \left(z_{0} \neq \infty\right), \\ 1 / \lim _{z \rightarrow \infty}\left(f^{\circ n}\right)^{\prime}(z) & \left(z_{0}=\infty\right) .\end{cases}
$$

Periodic points are classified as follows.

- The periodic point $z_{0}$ is superattracting if $\lambda=0$.

- The periodic point $z_{0}$ is attracting if $0<|\lambda|<1$.

- The periodic point $z_{0}$ is indifferent if $|\lambda|=1$.

- The periodic point $z_{0}$ is repelling if $|\lambda|>1$.

Indifferent periodic points are classified into the following two cases.

- The periodic point $z_{0}$ is parabolic if $\lambda$ is a root of unity.

- The periodic point $z_{0}$ is irrationally indifferent if $|\lambda|=1$ and $\lambda$ is not a root of unity.

In the case that the periodic point $z_{0}$ of period $n$ is superattracting or attracting, the attracting basin $\mathcal{A}\left(z_{0}\right)$ of $z_{0}$ is defined as

$$
\mathcal{A}\left(z_{0}\right)=\left\{z \in \hat{\mathbb{C}}: \lim _{k \rightarrow \infty} f^{\circ k n}(z)=z_{0}\right\}
$$

Every superattracting and attracting periodic point belongs to the Fatou set, and every parabolic and repelling periodic point belongs to the Julia set. Attracting basins of periodic points are subsets of the Fatou set. It is difficult to distinguish whether an irrationally indifferent periodic point belongs to the Fatou set or the Julia set. The Julia set is characterized by repelling periodic points.

Theorem 2.3. The Julia set $\mathcal{J}(f)$ is equal to the closure of the set of all repelling periodic points of $f$.

We investigate dynamics of the Blaschke product

$$
B(z)=e^{2 \pi i \theta} z \frac{z-a_{1}}{1-\overline{a_{1}} z} \cdots \frac{z-a_{\nu}}{1-\overline{a_{\nu}} z}=e^{2 \pi i \theta} z b_{1}(z) \cdots b_{\nu}(z)
$$


and its conjugate $A=\phi \circ B \circ \phi^{-1}$, where $\theta \in[0,1)$ is a real parameter, $a_{j} \in \mathbb{D}$ is a complex parameter $(1 \leq j \leq \nu)$ and

$$
b_{j}(z)=\frac{z-a_{j}}{1-\overline{a_{j}} z}, \quad \phi(z)=i \frac{z+1}{z-1} .
$$

It is easy to check that the Möbius transformation $b_{j}$ maps the unit disk onto itself and the Möbius transformation $\phi$ maps the unit disk onto the lower half-plane.

Lemma 2.4. The Blaschke product $B$ has attracting fixed points at the origin and the point at infinity.

Proof. Fixed points of $B$ are the solutions of the equation $B(z)=z$ or

$$
z\left[e^{2 \pi i \theta} b_{1}(z) \cdots b_{\nu}(z)-1\right]=0 .
$$

Since the Möbius transformation $b_{j}$ maps the unit disk onto itself, the inequality

$$
\left|e^{2 \pi i \theta} b_{1}(z) \cdots b_{\nu}(z)\right|<1
$$

holds for any $z \in \mathbb{D}$. Therefore, there are no fixed points of $B$ in the unit disk except for the origin. The derivative of $B$ is that

$$
B^{\prime}(z)=e^{2 \pi i \theta} b_{1}(z) \cdots b_{\nu}(z)+e^{2 \pi i \theta} z \sum_{j=1}^{\nu} b_{1}(z) \cdots b_{j}^{\prime}(z) \cdots b_{\nu}(z)
$$

and the multiplier at the origin is that

$$
B^{\prime}(0)=e^{2 \pi i \theta} b_{1}(0) \cdots b_{\nu}(0)=(-1)^{\nu} e^{2 \pi i \theta} a_{1} \cdots a_{\nu} .
$$

Since $\left|B^{\prime}(0)\right|<1$, the origin is an attracting fixed point. The Blaschke product $B$ is conjugate to itself via $\iota: z \mapsto 1 / \bar{z}$. This indicates that there are no fixed points of $B$ in $\hat{\mathbb{C}} \backslash \overline{\mathbb{D}}$ except for the point at infinity. The multiplier $\lambda$ at $\infty$ is that

$$
\lambda=\frac{1}{\lim _{z \rightarrow \infty} B^{\prime}(z)}=(-1)^{\nu} e^{-2 \pi i \theta} \overline{a_{1}} \cdots \overline{a_{\nu}} .
$$

Since $|\lambda|<1$, the point at infinity is also an attracting fixed point.

Lemma 2.5. The rational function $A=\phi \circ B \circ \phi^{-1}$ has attracting fixed points at $\pm i$.

Proof. Since $\phi(\infty)=i$ and $\phi(0)=-i$, the result follows by the above lemma.

Lemma 2.6. The Julia set $\mathcal{J}(B)$ is the unit circle $S^{1}$. 
Proof. The Möbius transformation $b_{j}$ maps the unit disk onto itself. Moreover, $b_{j}$ maps $S^{1}$ and $\hat{\mathbb{C}} \backslash \overline{\mathbb{D}}$ onto themselves respectively. This indicates that the unit circle $S^{1}$ is completely invariant under $B$, namely

$$
B\left(S^{1}\right)=S^{1}=B^{-1}\left(S^{1}\right) .
$$

Since the Julia set $\mathcal{J}(B)$ is the smallest closed completely invariant set containing at least three points, the Julia set $\mathcal{J}(B)$ is contained in the unit circle $S^{1}$ or

$$
\mathcal{J}(B) \subset S^{1}
$$

We prove that $\mathcal{J}(B)=S^{1}$ in the rest of the proof. We assume that there exists a point $\zeta$ which is in $S^{1} \backslash \mathcal{J}(B)$, namely $\zeta$ is in $\mathcal{F}(B)$. In this case, the Fatou set $\mathcal{F}(B)$ is the union of two attracting basins $\mathcal{A}(0)$ and $\mathcal{A}(\infty)$. Hence, the orbit of $\zeta$ tends to the attracting fixed point 0 or $\infty$. On the other hand, the orbit of $\zeta$ stays on the unit circle $S^{1}$ since it is invariant under $B$. This is a contradiction. Therefore, we obtain that $\mathcal{J}(B)=S^{1}$.

Lemma 2.7. The Julia set $\mathcal{J}(A)$ is the extended real line $\mathbb{R} \cup\{\infty\}$.

Proof. Since $A$ is conjugate to $B$ via $\phi$ or $A=\phi \circ B \circ \phi^{-1}$, we obtain that

$$
\mathcal{J}(A)=\phi(\mathcal{J}(B))=\phi\left(S^{1}\right)=\mathbb{R} \cup\{\infty\}
$$

Lemma 2.8. The equation

$$
b_{j} \circ \phi^{-1}(z)=\frac{1-a_{j}}{1-\overline{a_{j}}} \cdot \frac{z+i a_{j}^{*}}{z-i \overline{a_{j}^{*}}}
$$

holds, where

$$
a_{j}^{*}=\frac{1+a_{j}}{1-a_{j}} .
$$

Proof.

$$
\begin{aligned}
b_{j} \circ \phi^{-1}(z)=b_{j}\left(\frac{z+i}{z-i}\right) & =\frac{(z+i)-a_{j}(z-i)}{(z-i)-\overline{a_{j}}(z+i)}=\frac{\left(1-a_{j}\right) z+i\left(1+a_{j}\right)}{\left(1-\overline{a_{j}}\right) z-i\left(1+\overline{a_{j}}\right)} \\
& =\frac{1-a_{j}}{1-\overline{a_{j}}} \cdot \frac{z+i \frac{1+a_{j}}{1-a_{j}}}{z-i \frac{1+\overline{a_{j}}}{1-\overline{a_{j}}}}=\frac{1-a_{j}}{1-\overline{a_{j}}} \cdot \frac{z+i a_{j}^{*}}{z-i \overline{a_{j}^{*}}}
\end{aligned}
$$


We calculate exact form of the rational function $A$. By the above lemma,

$$
B \circ \phi^{-1}(z)=e^{2 \pi i \theta} \phi^{-1}(z) \prod_{j=1}^{\nu} b_{j}\left(\phi^{-1}(z)\right)=e^{2 \pi i \theta} \frac{z+i}{z-i} \prod_{j=1}^{\nu} \frac{1-a_{j}}{1-\overline{a_{j}}} \cdot \frac{z+i a_{j}^{*}}{z-i \overline{a_{j}^{*}}} .
$$

Therefore, we obtain that

$$
\begin{aligned}
A(z) & =\phi \circ B \circ \phi^{-1}(z) \\
= & i \frac{e^{2 \pi i \theta} \frac{z+i}{z-i} \prod_{j=1}^{\nu} \frac{1-a_{j}}{1-\overline{a_{j}}} \cdot \frac{z+i \bar{a}_{j}^{*}}{z-i \overline{a_{j}^{*}}}+1}{e^{2 \pi i \theta} \frac{z+i}{z-i} \prod_{j=1}^{\nu} \frac{1-a_{j}}{1-\overline{a_{j}}} \cdot \frac{z+i a_{j}^{*}}{z-i \overline{a_{j}^{*}}}-1} \\
= & i \frac{e^{\pi i \theta}(z+i) \prod_{j=1}^{\nu}\left(1-a_{j}\right)\left(z+i a_{j}^{*}\right)+e^{-\pi i \theta}(z-i) \prod_{j=1}^{\nu}\left(1-\overline{a_{j}}\right)\left(z-i \overline{a_{j}^{*}}\right)}{e^{\pi i \theta}(z+i) \prod_{j=1}^{\nu}\left(1-a_{j}\right)\left(z+i a_{j}^{*}\right)-e^{-\pi i \theta}(z-i) \prod_{j=1}^{\nu}\left(1-\overline{a_{j}}\right)\left(z-i \overline{a_{j}^{*}}\right)} \\
= & i \frac{A_{1}(z)+A_{2}(z)}{A_{1}(z)-A_{2}(z)},
\end{aligned}
$$

where

$$
A_{1}(z)=e^{\pi i \theta}(z+i) \prod_{j=1}^{\nu}\left(1-a_{j}\right)\left(z+i a_{j}^{*}\right), \quad A_{2}(z)=e^{-\pi i \theta}(z-i) \prod_{j=1}^{\nu}\left(1-\overline{a_{j}}\right)\left(z-i \overline{a_{j}^{*}}\right) .
$$

Proposition 2.9. $\overline{A_{1}(x)}=A_{2}(x)$ and $\overline{A_{1}^{\prime}(x)}=A_{2}^{\prime}(x)$ hold for all $x \in \mathbb{R}$. Moreover, the following inequality holds for all $x \in \mathbb{R}$ :

$$
A^{\prime}(x)=\frac{\operatorname{Im}\left[A_{1}(x) A_{2}^{\prime}(x)\right]}{\left[\operatorname{Im} A_{1}(x)\right]^{2}}>0 .
$$

Proof. The first two equations are obvious. The derivative of $A$ is that

$$
A^{\prime}(z)=i \frac{2\left[A_{1}(z) A_{2}^{\prime}(z)-A_{1}^{\prime}(z) A_{2}(z)\right]}{\left[A_{1}(z)-A_{2}(z)\right]^{2}} .
$$

We transform the numerator and the denominator of the above equation as $A_{1}(x) A_{2}^{\prime}(x)-A_{1}^{\prime}(x) A_{2}(x)=A_{1}(x) A_{2}^{\prime}(x)-\overline{A_{2}^{\prime}(x) A_{1}(x)}=2 i \operatorname{Im}\left[A_{1}(x) A_{2}^{\prime}(x)\right]$ and

$$
A_{1}(x)-A_{2}(x)=A_{1}(x)-\overline{A_{1}(x)}=2 i \operatorname{Im} A_{1}(x)
$$


for all $x \in \mathbb{R}$. Therefore, we obtain that

$$
A^{\prime}(x)=i \frac{2 \cdot 2 i \operatorname{Im}\left[A_{1}(x) A_{2}^{\prime}(x)\right]}{\left[2 i \operatorname{Im} A_{1}(x)\right]^{2}}=\frac{\operatorname{Im}\left[A_{1}(x) A_{2}^{\prime}(x)\right]}{\left[\operatorname{Im} A_{1}(x)\right]^{2}}
$$

for all $x \in \mathbb{R}$. We calculate the numerator of the last equation.

$$
\begin{aligned}
& A_{1}(x) A_{2}^{\prime}(x) \\
= & e^{\pi i \theta}(x+i) \prod_{j=1}^{\nu}\left(1-a_{j}\right)\left(x+i a_{j}^{*}\right) \\
& \times e^{-\pi i \theta}\left[\prod_{j=1}^{\nu}\left(1-\overline{a_{j}}\right)\left(x-i \overline{a_{j}^{*}}\right)+(x-i) \prod_{j=1}^{\nu}\left(1-\overline{a_{j}}\right) \cdot \sum_{j=1}^{\nu} \prod_{k \neq j}\left(x-i \overline{a_{k}^{*}}\right)\right] \\
= & (x+i) \prod_{j=1}^{\nu}\left|1-a_{j}\right|^{2}\left|x+i a_{j}^{*}\right|^{2}+|x+i|^{2}\left(\prod_{j=1}^{\nu}\left|1-a_{j}\right|^{2}\right) \sum_{j=1}^{\nu}\left(x+i a_{j}^{*}\right) \prod_{k \neq j}\left|x+i a_{k}^{*}\right|^{2} .
\end{aligned}
$$

Since

$x+i a_{j}^{*}=x+i \frac{1+a_{j}}{1-a_{j}}=x+i \frac{1-\left(\alpha_{j}^{2}+\beta_{j}^{2}\right)+i \cdot 2 \beta_{j}}{\left(1-\alpha_{j}\right)^{2}+\beta_{j}^{2}} \quad\left(a_{j}=\alpha_{j}+i \beta_{j} \in \mathbb{D}\right)$

and

$$
\operatorname{Im}\left(x+i a_{j}^{*}\right)=\frac{1-\left(\alpha_{j}^{2}+\beta_{j}^{2}\right)}{\left(1-\alpha_{j}\right)^{2}+\beta_{j}^{2}}>0,
$$

we obtain that

$$
\begin{aligned}
\operatorname{Im}\left[A_{1}(x) A_{2}^{\prime}(x)\right] & =\prod_{j=1}^{\nu}\left|1-a_{j}\right|^{2}\left|x+i a_{j}^{*}\right|^{2} \\
& +|x+i|^{2}\left(\prod_{j=1}^{\nu}\left|1-a_{j}\right|^{2}\right) \sum_{j=1}^{\nu} \frac{1-\left(\alpha_{j}^{2}+\beta_{j}^{2}\right)}{\left(1-\alpha_{j}\right)^{2}+\beta_{j}^{2}} \prod_{k \neq j}\left|x+i a_{k}^{*}\right|^{2}>0 .
\end{aligned}
$$

Therefore, the inequality

$$
A^{\prime}(x)=\frac{\operatorname{Im}\left[A_{1}(x) A_{2}^{\prime}(x)\right]}{\left[\operatorname{Im} A_{1}(x)\right]^{2}}>0
$$

holds for all $x \in \mathbb{R}$.

Corollary 2.10. The multiplier of any repelling periodic orbit of $A$

$$
\zeta_{1} \mapsto \zeta_{2} \mapsto \cdots \mapsto \zeta_{p} \mapsto \zeta_{1}
$$

is greater than one. 
Proof. We may assume that $p=1$. Let $\zeta \in \mathcal{J}(A)=\mathbb{R} \cup\{\infty\}$ be a repelling fixed point. Its multiplier $\mu$ satisfies that $|\mu|>1$ and

$$
\mu= \begin{cases}A^{\prime}(\zeta)>0 & (\zeta \neq \infty) \\ 1 / \lim _{x \rightarrow \infty} A^{\prime}(x) \geq 0 & (\zeta=\infty)\end{cases}
$$

Therefore, we obtain that $\mu>1$.

\section{Configurations of equilibrium points}

The proof of the main theorem relies on Lemma 3.4 which determines configurations of equilibrium points and poles. The main ingredient of the proof of Lemma 3.4 is the Euler-Jacobi formula.

Theorem 3.1 (The Euler-Jacobi Formula). Let $f: \mathbb{C} \rightarrow \mathbb{C}$ be a polynomial of degree $d$. If all zeros $w_{1}, w_{2}, \ldots, w_{d}$ of $f$ are simple, then

$$
\sum_{j=1}^{d} \frac{g\left(w_{j}\right)}{f^{\prime}\left(w_{j}\right)}=0
$$

for any polynomial $g$ satisfying that $\operatorname{deg}(g)<\operatorname{deg}\left(f^{\prime}\right)=d-1$.

Proof. We consider the rational function $g(z) / f(z)$. Let $\Gamma$ be a circle with a large radius $r$ surrounding all zeros of $f$. Applying the residues theorem, we obtain that

$$
\int_{\Gamma} \frac{g(z)}{f(z)} d z=2 \pi i \sum_{j=1}^{d} \operatorname{Res}\left(\frac{g}{f}, w_{j}\right)=2 \pi i \sum_{j=1}^{d} \frac{g\left(w_{j}\right)}{f^{\prime}\left(w_{j}\right)}
$$

On the other hand, we obtain that

$$
\left|\int_{\Gamma} \frac{g(z)}{f(z)} d z\right| \leq 2 \pi r \max _{|z|=r}\left|\frac{g(z)}{f(z)}\right|
$$

Since $\operatorname{deg}(g)+1<\operatorname{deg}(f)=d$, the right hand side of the inequality tends to zero as the radius $r$ tends to infinity.

There are some applications of the Euler-Jacobi formula in [1].

Proposition 3.2 ([1, Proposition 2.6]). Let $f$ be a polynomial of degree $d \geq$ 2. We consider the differential equation (DE) and assume that all equilibrium points $w_{1}, w_{2}, \ldots, w_{d}$ of $(\mathrm{DE})$ are simple, namely all zeros $w_{1}, w_{2}, \ldots, w_{d}$ of $f$ are simple. Then the following statements hold. 
(a) If $w_{1}, w_{2}, \ldots, w_{d-1}$ are nodes, then $w_{d}$ is also a node.

(b) If $w_{1}, w_{2}, \ldots, w_{d-1}$ are centers, then $w_{d}$ is also a center.

(c) If not all equilibrium points are centers, then there exist at least two of them that have different stability.

Proposition 3.3 ([1, Proposition 2.7]). Let $f$ be a polynomial of degree d. We consider the differential equation (DE) and assume that all equilibrium points of (DE) are simple. Moreover, we assume that $d-2 k$ equilibrium points $z_{1}, \ldots, z_{d-2 k}$ are located on a straight line $\mathcal{L}$ for some $k \geq 0$ and the other $2 k$ equilibrium points $z_{d-2 k+1}, \ldots, z_{d}$ are symmetric with respect to the line $\mathcal{L}$. Then the following statements hold.

(a) All the points on $\mathcal{L}$ are of the same type and if they are not centers, then they have alternated stability.

(b) If all the points on $\mathcal{L}$ are of center type, then each pair of symmetric points with respect to $\mathcal{L}$ is formed by two points of the same type and if they are not centers, then they have opposite stability.

(c) If all the points on $\mathcal{L}$ are of node type, then each pair of symmetric points with respect to $\mathcal{L}$ is formed by two points of the same type and if they are not centers, then they have the same stability.

Theorem 1.2 and the main theorem are motivated by Proposition 3.3. If all simple equilibrium points of the "polynomial" differential equation (DE) are located on a straight line and they are not centers, then they have alternated stability (Proposition 3.3.a). Theorem 1.2 and the main theorem are counterexamples in the case that $f$ is a genuine rational function.

Lemma 3.4. Let $F(z)=P(z) / Q(z)$ be a rational function, where $P$ and $Q$ are polynomials with no common factors and of degrees $\operatorname{deg}(P)=n$ and $\operatorname{deg}(Q)=m$ respectively. We suppose that the two polynomials $P$ and $Q$ have only simple roots. Let $s_{1}, s_{2}, \ldots, s_{n-2 k}$ be the real zeros of $P$ with the order $s_{n-2 k}<\cdots<s_{2}<s_{1}$, and let $w_{1}, \overline{w_{1}}, \ldots, w_{k}, \overline{w_{k}}$ be the other zeros of $P$ with $\operatorname{Im}\left(w_{j}\right) \neq 0$. Then the equation

$$
F^{\prime}\left(s_{\delta}\right)=-\frac{Q\left(s_{\gamma}\right)}{Q\left(s_{\delta}\right)} \cdot \frac{R_{\gamma \delta}\left(s_{\delta}\right)}{R_{\gamma \delta}\left(s_{\gamma}\right)} \cdot F^{\prime}\left(s_{\gamma}\right)
$$

holds, where $\gamma<\delta$ and

$$
R_{\gamma \delta}(z)=\prod_{j \neq \gamma, \delta}\left(z-s_{j}\right) \prod_{j=1}^{k}\left(z-w_{j}\right)\left(z-\overline{w_{j}}\right) .
$$


If $\delta-\gamma$ is odd, then $R_{\gamma \delta}\left(s_{\gamma}\right)$ and $R_{\gamma \delta}\left(s_{\delta}\right)$ have the same sign or

$$
R_{\gamma \delta}\left(s_{\gamma}\right) \cdot R_{\gamma \delta}\left(s_{\delta}\right)>0
$$

Proof. The derivative of the function $F$ is that

$$
F^{\prime}(z)=\frac{P^{\prime}(z) Q(z)-P(z) Q^{\prime}(z)}{Q(z)^{2}}=\frac{P^{\prime}(z)}{Q(z)}-\frac{P(z) Q^{\prime}(z)}{Q(z)^{2}}
$$

and we obtain that

$$
F^{\prime}\left(z_{j}\right)=\frac{P^{\prime}\left(z_{j}\right)}{Q\left(z_{j}\right)}
$$

for any zero $z_{j}$ of $P$. The polynomial $P$ has the form

$$
P(z)=C \prod_{j=1}^{n}\left(z-z_{j}\right)=C \prod_{j=1}^{n-2 k}\left(z-s_{j}\right) \prod_{j=1}^{k}\left(z-w_{j}\right)\left(z-\overline{w_{j}}\right)
$$

where $C$ is a constant. Applying the Euler-Jacobi formula to the rational function $R_{\gamma \delta}(z) / P(z)$, we obtain that

$$
\sum_{j=1}^{n} \frac{R_{\gamma \delta}\left(z_{j}\right)}{P\left(z_{j}\right)}=\frac{R_{\gamma \delta}\left(s_{\gamma}\right)}{P^{\prime}\left(s_{\gamma}\right)}+\frac{R_{\gamma \delta}\left(s_{\delta}\right)}{P^{\prime}\left(s_{\delta}\right)}=0
$$

or

$$
P^{\prime}\left(s_{\delta}\right)=-\frac{R_{\gamma \delta}\left(s_{\delta}\right)}{R_{\gamma \delta}\left(s_{\gamma}\right)} \cdot P^{\prime}\left(s_{\gamma}\right) .
$$

We transform the last equation as

$$
\frac{P^{\prime}\left(s_{\delta}\right)}{Q\left(s_{\delta}\right)}=-\frac{Q\left(s_{\gamma}\right)}{Q\left(s_{\delta}\right)} \cdot \frac{R_{\gamma \delta}\left(s_{\delta}\right)}{R_{\gamma \delta}\left(s_{\gamma}\right)} \cdot \frac{P^{\prime}\left(s_{\gamma}\right)}{Q\left(s_{\gamma}\right)}
$$

and we obtain that

$$
F^{\prime}\left(s_{\delta}\right)=-\frac{Q\left(s_{\gamma}\right)}{Q\left(s_{\delta}\right)} \cdot \frac{R_{\gamma \delta}\left(s_{\delta}\right)}{R_{\gamma \delta}\left(s_{\gamma}\right)} \cdot F^{\prime}\left(s_{\gamma}\right)
$$

In the case that $\delta-\gamma$ is odd, the statement is obvious.

Proof of the Main Theorem. Equilibrium points of the differential equation $(\mathrm{DE}: \boldsymbol{a} ; \theta ; n)$ correspond to periodic points of period $k(k \mid n)$ of $A$ or fixed points of $A^{\text {on }}$. Let

$$
\left\{s_{j}^{\langle n\rangle}\right\}_{j=1}^{(\nu+1)^{n}-\varepsilon} \subset \mathcal{E P}\left(A^{* n}\right)
$$

be the set of all fixed points of $A^{\circ n}$ in $\mathbb{C}$ except attracting fixed points $\pm i$, where $\varepsilon=2$ if $\infty$ is a fixed point of $A^{\circ n}$ or $\varepsilon=1$ if $\infty$ is not a fixed point of $A^{\text {on }}$. 
(a) By Lemma 2.7, there are no attracting or parabolic periodic points of $A$ except attracting fixed points $\pm i$. Hence, each $s_{j}^{\langle n\rangle}$ is a repelling fixed point of $A^{\text {on }}$ or

$$
\left\{s_{j}^{\langle n\rangle}\right\}_{j=1}^{(\nu+1)^{n}-\varepsilon} \subset \mathcal{J}(A) .
$$

Let $\lambda_{ \pm}$be the multiplier at $\pm i$. Since $\left|\lambda_{ \pm}\right|<1$ and

$$
\operatorname{Re}\left(A^{* n}\right)^{\prime}( \pm i)=\operatorname{Re}\left(\lambda_{ \pm}\right)^{n}-1<0
$$

equilibrium points $\pm i$ are stable nodes or stable foci.

(b) Since

$\left\{s_{j}^{\langle n\rangle}\right\}_{j=1}^{(\nu+1)^{n}-\varepsilon} \subset \mathcal{J}(A)=\mathbb{R} \cup\{\infty\}$ and $\mathcal{E P}\left(A^{* n}\right)=\left\{s_{j}^{\langle n\rangle}\right\}_{j=1}^{(\nu+1)^{n}-\varepsilon} \cup\{ \pm i\}$,

all equilibrium points of the differential equation (DE : $\boldsymbol{a} ; \theta ; n)$ are symmetric with respect to the real axis.

(c) Let $\mu$ be the multiplier of $s_{j}^{\langle n\rangle}$. By Corollary 2.10,

$$
\left(A^{* n}\right)^{\prime}\left(s_{j}^{\langle n\rangle}\right)=\mu^{\frac{n}{k}}-1>0
$$

Therefore, the equilibrium point $s_{j}^{\langle n\rangle}$ is an unstable node.

(d) We assume that

$$
s_{(\nu+1)^{n}-\varepsilon}^{\langle n\rangle}<\cdots<s_{j+1}^{\langle n\rangle}<s_{j}^{\langle n\rangle}<\cdots<s_{1}^{\langle n\rangle} .
$$

The rational function $A^{* n}$ can be written as

$$
A^{* n}(z)=\frac{P^{\langle n\rangle}(z)}{Q^{\langle n\rangle}(z)},
$$

where $P^{\langle n\rangle}$ and $Q^{\langle n\rangle}$ are polynomials with no common roots. Then equilibrium points of the differential equation (DE : $\boldsymbol{a} ; \theta ; n)$ correspond to zeros of $P^{\langle n\rangle}$ and poles of $A^{* n}$ correspond to zeros of $Q^{\langle n\rangle}$. Since the Julia set $\mathcal{J}(A)=\mathbb{R} \cup\{\infty\}$ is completely invariant under $A$, all poles of $A^{* n}$ in $\mathbb{C}$ belong to $\mathbb{R}$. Let

$$
\left\{t_{j}^{\langle n\rangle}\right\}_{j=1}^{(\nu+1)^{n}+1-\varepsilon}=\left(A^{* n}\right)^{-1}(\infty) \backslash\{\infty\} \subset \mathbb{R}
$$

be the set of all poles of $A^{* n}$ in $\mathbb{C}$, where

$$
t_{(\nu+1)^{n}+1-\varepsilon}^{\langle n\rangle}<\cdots<t_{j+1}^{\langle n\rangle}<t_{j}^{\langle n\rangle}<\cdots<t_{1}^{\langle n\rangle} .
$$


Then we can assume that

$$
P^{\langle n\rangle}(z)=C_{n} \prod_{j=1}^{(\nu+1)^{n}-\varepsilon}\left(z-s_{j}^{\langle n\rangle}\right) \times(z+i)(z-i)
$$

and

$$
Q^{\langle n\rangle}(z)=\prod_{j=1}^{(\nu+1)^{n}+1-\varepsilon}\left(z-t_{j}^{\langle n\rangle}\right)
$$

where $C_{n}$ is a constant. We apply Lemma 3.4 to $F=A^{* n}$ for $\gamma=j$ and $\delta=j+1$. Let

$$
R_{j(j+1)}(z)=\prod_{k \neq j, j+1}\left(z-s_{k}^{\langle n\rangle}\right) \times(z+i)(z-i) .
$$

Since $s_{j}^{\langle n\rangle}$ and $s_{j+1}^{\langle n\rangle}$ are unstable nodes,

$$
\left(A^{* n}\right)^{\prime}\left(s_{j}^{\langle n\rangle}\right)>0 \text { and }\left(A^{* n}\right)^{\prime}\left(s_{j+1}^{\langle n\rangle}\right)>0 .
$$

By Lemma 3.4,

$$
R_{j(j+1)}\left(s_{j}^{\langle n\rangle}\right) \cdot R_{j(j+1)}\left(s_{j+1}^{\langle n\rangle}\right)>0 .
$$

Therefore, we obtain that

$$
Q^{\langle n\rangle}\left(s_{j}^{\langle n\rangle}\right) \cdot Q^{\langle n\rangle}\left(s_{j+1}^{\langle n\rangle}\right)<0 .
$$

The last inequality indicates that the number of poles between equilibrium points $s_{j}^{\langle n\rangle}$ and $s_{j+1}^{\langle n\rangle}$ is odd for all $j \geq 1$. Since the number of equilibrium points of the differential equation (DE: $\boldsymbol{a} ; \theta ; n)$ on the real axis is $(\nu+1)^{n}-\varepsilon$ and the number of poles of $A^{* n}$ is $(\nu+1)^{n}+1-\varepsilon$, there is just one pole between equilibrium points $s_{j}^{\langle n\rangle}$ and $s_{j+1}^{\langle n\rangle}$ for all $j \geq 1$, namely the following inequality holds:

$t_{(\nu+1)^{n}+1-\varepsilon}^{\langle n\rangle}<s_{(\nu+1)^{n}-\varepsilon}^{\langle n\rangle}<t_{(\nu+1)^{n}-\varepsilon}^{\langle n\rangle}<\cdots<s_{j}^{\langle n\rangle}<t_{j}^{\langle n\rangle}<\cdots<t_{2}^{\langle n\rangle}<s_{1}^{\langle n\rangle}<t_{1}^{\langle n\rangle}$.

Therefore, equilibrium points of the differential equation (DE: $\boldsymbol{a} ; \theta ; n)$ on the real axis and poles of $A^{* n}$ are located alternately.

\section{References}

[1] M. J. Álvarez, A. Gasull and R. Prohens, Configurations of critical points in complex polynomial differential equations, Nonlinear Anal. 71 (2009), 923-934. http://dx.doi.org/10.1016/j.na.2008.11.018 
[2] M. J. Álvarez, A. Gasull and R. Prohens, Topological classification of polynomial complex differential equations with all the critical points of center type, J. Difference Equ. Appl. 16 (2010), 411-423.

http://dx.doi.org/10.1080/10236190903232654

[3] K. Katagata, Qualitative Theory of Differential Equations and Dynamics of Quadratic Rational Functions, Nonl. Analysis and Differential Equations 2 (2014), 45-59. http://dx.doi.org/10.12988/nade.2014.3819

[4] J. Milnor, Dynamics in One Complex Variable, Vieweg, 2nd edition, 2000. http://dx.doi.org/10.1007/978-3-663-08092-3

Received: October 11, 2014; Published: December 3, 2014 